\title{
Anisotropic clustering of inertial particles in homogeneous shear flow
}

\author{
P. Gualtieri, F. Picano, C.M. Casciola \\ Dipartimento di Meccanica e Aeronautica, \\ Università di Roma La Sapienza \\ Via Eudossiana 18, 00184 Roma Italy
}

\begin{abstract}
Recently, clustering of inertial particles in turbulence has been thoroughly analyzed for statistically homogeneous isotropic flows. Phenomenologically, spatial homogeneity of particles configurations is broken by the advection of a range of eddies determined by the Stokes relaxation time of the particles which results in a multi-scale distribution of local concentrations and voids. Much less is known concerning anisotropic flows. Here, by addressing direct numerical simulations (DNS) of a statistically steady particle-laden homogeneous shear flow, we provide evidence that the mean shear preferentially orients particle patterns. By imprinting anisotropy on large scales velocity fluctuations, the shear indirectly affects the geometry of the clusters. Quantitative evaluation is provided by a purposely designed tool, the angular distribution function of particle pairs (ADF), which allows to address the anisotropy content of particles aggregates on a scale by scale basis. The data provide evidence that, depending on the Stokes relaxation time of the particles, anisotropic clustering may occur even in the range of scales where the carrier phase velocity field is already recovering isotropy. The strength of the singularity in the anisotropic component of the ADF quantifies the level of fine scale anisotropy, which may even reach values of more than $30 \%$ direction-dependent variation in the probability to find two close-by particles at viscous scale separation.
\end{abstract}

\section{Introduction}

Transport of inertial particles is involved in several fields of science, e.g. droplets growth in clouds, (1) 2), planetary formations, (3), or plankton 
accumulation in the ocean, (4). As far as technological applications are concerned, inertial particle dynamics is crucial for solid or liquid fuelled rockets, injection systems of internal combustion engines or for sediments accumulation in pipelines, e.g. (5; 6). Inertial particles differ from perfectly Lagrangian tracers due to inertia which prevents them from following the flow trajectories. The main effect consists of "preferential accumulation", see for instance (7; 5). In homogeneous isotropic conditions it amounts to the small-scale clustering discussed in a number of recent papers, (8; ; , 10).

In presence of inhomogeneity new features emerge leading to the socalled turbophoresis as a preferential accumulation near the boundary in wall turbulence (11). Under appropriate conditions, particles may achieve extremely large concentrations at the wall with a substantial reduction of mobility. This turbulence-induced transport and the issuing preferential accumulation as been addressed in a number of papers dealing with a variety of configurations, from boundary layers to planar channels and pipes, attacked both from the experimental $(12$; 13 , 14) and the numerical (15; 6, 15) side. Though a complete understanding of the phenomenon is still lacking, the advection of the particles by the coherent motions in the wall-layer is certainly essential, as discussed by (16). In other words the structures responsible for particle accumulation at the wall are the same which sustain turbulence kinetic energy production in the buffer layer, see also (5; 6). Turbophoresis and small scale clustering are different aspects of the same inertial particle dynamics. Both phenomena are induced by non trivial phase relationships due to quasi-coherent vortical structures. The main difference is provided by the characteristic scales, associated with the Kolmogorov time unit $\tau_{\eta}=\eta^{2} / \nu$ in one case $-\eta$ being Kolmogorov length and $\nu$ the kinematic viscosity-and with the larger energy producing time scale in the other. Inhomogeneity is essential to have spatial segregation. For instance, in the kinetic model presented in (11), the spatial transport of particle concentration presents, beside a Fick-like gradient type diffusion component, a contribution associated with the spatial variations of turbulence intensity. However anisotropy is probably a key ingredient of the process, see e.g. the preferential direction of the trajectories of particles approaching the wall (16). The two features are strongly entangled in wall bounded flows. A special flow exists however-the homogeneous shear flow in a confined boxwhich retains most of the anisotropic dynamics of wall bounded flows still preserving, on average, spatial homogeneity.

The flow is bound by a computational box of fixed extension and its integral scale grows initially to eventually saturate due to confinement. Target of the analysis is the statically steady state with time independent ensemble 
averages. Similar features are found in the the experimental data by (17). By using an active grid to generate a flow with integral scale close to the transversal dimension of the apparatus, the authors were able to achieve confinement from the outstart. As a consequence, the integral scale did not increase downstream, see also the discussion in (18).

Our flow shares with the wall-layer streamwise vortices and turbulent kinetic energy production mechanisms. In the numerical experiment, this corresponds to pseudo-cyclic fluctuations associated with the regeneration of streamwise vortical structures.

Velocity fluctuations are strongly anisotropic at the large scales driven by production while, for smaller separations, the classical energy transfer mechanisms become effective in inducing re-isotropization. This classical issue, see e.g. (19), has been recently revisited by more complete diagnostic tools (e.g. $\mathrm{SO}(3)$ decomposition of turbulent fluctuations) able to quantify on a scale-by-scale basis the amount of anisotropy in the carrier fluid, as discussed both experimentally $(20 ; 21)$ and numerically $(22)$ (see $(23)$ for a review).

Despite anisotropy of the velocity field is now well understood and the carrier fluid shows tendency towards isotropy recovery below the shear scale, $L_{\mathrm{S}}=\sqrt{\epsilon / S^{3}}$ with $\epsilon$ the average turbulent kinetic energy dissipation rate per unit mass and $S$ the average shear rate, the behavior of particle distributions is still not fully explored.

Anisotropic transport of inertial particles has been recently addressed by (24), who analyzed numerically the initial transient of the homogeneous shear flow, i.e. before saturation of the integral scale occurs, with the purpose of modeling unconfined conditions. The focus was mainly on the comparison of different particle dynamics models. However, by considering particle configurations in orthogonal planes, the authors also discussed the anisotropy of particle clusters concluding that particles are most concentrated in the streamwise and least concentrated in the cross-stream direction. The same flow was dealt with by $(25,26$; 27$)$ to investigate issues such as turbulence modulation in the two way coupling regime or heat transfer induced by the disperse phase.

Purpose of the present paper is the quantitative evaluation of the shear induced anisotropy in particles clustering. It is now well known that particles respond to the fluid velocity fluctuations in a certain range of scales which is determined by their Stokes time. The relevant parameter is the Stokes number, ratio of particle Stokes time and flow time scale. In order to work with well defined conditions, one needs a shear flow whose characteristic time and length scales are constant in time. The best candidate, is 
the statistically steady homogeneous shear flow in a confined box we have described above. In this flow, below the shear scale, the velocity fluctuations tend to recover isotropy. The question is then what happens to particle clusters. Do they become isotropic in the smallest scales? For given velocity field, how is the geometry of the clusters affected by the relaxation time of the particles?

In fact, the main contribution of the present study is the quantitative evidence that particle distributions do not necessarily reduce their anisotropy at small scales, despite the isotropy recovery occurring in the velocity field. Rather clusters anisotropy may even grow below the Kolmogorov length where the velocity field is smooth and almost isotropic. As a matter of fact, inertia manifest itself in a rather peculiar and unexpected way, and leads, under certain coupling conditions, to singular particle distributions which viscosity cannot regularize (8). After introducing a suitable observable-the angular distribution function- its spherical decomposition is used to evaluate the relative importance of its different components. The scaling exponents of the respective singularities show that, under appropriate conditions, anisotropy is a leading order effect which may easily persist down to vanishing separations. The data offer preliminary evidence of the anisotropic geometry of the fractal support of inertial particle distributions under shear flows, thus non-trivially extending results recently achieved for isotropic transport.

\section{Methodology}

Concerning the carrier fluid, the velocity field $\mathbf{v}$ is decomposed into a mean flow $\mathbf{U}=S x_{2} \mathbf{e}_{1}$ and a fluctuation $\mathbf{u}$, see figure 1 for notations. Rogallo's technique is employed to rewrite the Navier-stokes equations for velocity fluctuations in a deforming coordinate system convected by the mean flow according to the transformation of variables $\xi_{1}=x_{1}-S t x_{2} ; \quad \xi_{2}=$ $x_{2} ; \quad \xi_{3}=x_{3} ; \quad \tau=t,(28)$. The resulting system is numerically integrated by a pseudo-spectral method combined with a fourth order Runge-Kutta scheme for temporal evolution, see (29).

The two parameters controlling the homogeneous shear flow are the Taylor-Reynolds number $\operatorname{Re}_{\lambda}=\sqrt{5 /(\nu \epsilon)}\left\langle u_{\alpha} u_{\alpha}\right\rangle$ and the shear strength $S^{*}=$ $S\left\langle u_{\alpha} u_{\alpha}\right\rangle / \epsilon$. For the simulations discussed below they are $\operatorname{Re}_{\lambda} \simeq 100$ and $S^{*} \simeq 7$, corresponding to a ratio of shear to Kolmogorov scale $L_{s} / \eta \simeq 35$. Navier-Stokes equations are integrated in a $4 \pi \times 2 \pi \times 2 \pi$ periodic box with a resolution of $256 \times 256 \times 128$ Fourier modes corresponding to $384 \times 384 \times 192$ 
collocation points in physical space due to the $3 / 2$ dealiasing rule. The Kolmogorov scale is $\eta=0.02$ which correspond to $K_{\max } \eta=3.1$ ensuring sufficient resoltion at small scales. Actually a well resolved velocity field is crucial to minimize numerical errors associated with the interpolation of the fluid velocity at particle positions which is necessary to advect the particles in the present mixed Eulerian-Lagrangian formulation, see e.g. (30, 31).

The disperse phase consists of diluted particles with mass density $\rho_{p}$ much larger than the carrier fluid $\rho_{f}$, assumed small enough to be modeled as material points. At this dilution self-interactions and the back reaction on the fluid are negligible, leaving the Stokes drag in the relative motion with the fluid as the only relevant force on each particle, (32). Accordingly, the equations for particles position $x_{i}^{p}(t)$ and velocity $v_{i}^{p}(t)$ read

$$
\begin{aligned}
& \frac{d x_{i}^{p}}{d t}=v_{i}^{p} \\
& \frac{d v_{i}^{p}}{d t}=\frac{1}{\tau_{p}}\left[v_{i}\left(x^{p}, t\right)-v_{i}^{p}(t)\right]
\end{aligned}
$$

where $v_{i}\left(x^{p}, t\right)$ is the instantaneous fluid velocity evaluated at $x_{i}^{p}(t)$ and $\tau_{p}=\rho_{p} d_{p}^{2} /\left(18 \nu \rho_{f}\right)$ is the Stokes relaxation time $\left(d_{p}\right.$ denotes the particle diameter). The particle dynamics is controlled by the ratio of $\tau_{p}$ to a characteristic flow time scale, typically the Kolmogorov time scale $\tau_{\eta}=\eta^{2} / \nu$, i.e. the relevant control parameter is the Stokes number $S t_{\eta}=\tau_{p} / \tau_{\eta}$. Particle velocities are decomposed as $v_{i}^{p}=U_{i}\left[x_{k}^{p}(t)\right]+u_{i}^{p}$ where $u_{i}^{p}$ denotes the particle velocity deviation with respect to the local mean flow of the carrier fluid. Eqs. (1) can be written as

$$
\begin{aligned}
& \frac{d x_{i}^{p}}{d t}=u_{i}^{p}+U_{i}\left(x^{p}\right) \\
& \frac{d u_{i}^{p}}{d t}=\frac{1}{\tau_{p}}\left[u_{i}\left(x^{p}, t\right)-u_{i}^{p}(t)\right]-\frac{d U_{i}}{d t},
\end{aligned}
$$

to be finally rearranged in Rogallo's computational space as (see also (24))

$$
\begin{aligned}
& \frac{d \xi_{i}^{p}}{d \tau}=u_{i}^{p}-S \tau u_{2}^{p} \delta_{i 1} \\
& \frac{d u_{i}^{p}}{d \tau}=\frac{1}{\tau_{p}}\left[u_{i}\left(\xi^{p}, \tau\right)-u_{i}^{p}(\tau)\right]-S u_{2}^{p} \delta_{i 1} .
\end{aligned}
$$

The particle equations are integrated by the same fourth order Runge-Kutta scheme used for the Navier-Stokes equations, with fluid velocity at particle 
positions evaluated by tri-linear interpolation. The accuracy of the interpolation scheme may be an issue. To assess its effect on the numerical results, we have preliminarily run two different simulations at half the resolution and smaller Reynolds number of the cases to be discussed in the main body of the paper. The two simulations employ two different interpolation schemes, namely a linear and a quadratic one. As always in turbulence, comparisons need to be made in terms of the relevant statistical observables. We anticipate that here we deal with the different projections of the Angular Distribution Function, to be introduced in $\S 3$. In figure 2 the solid lines denote results obtained with the linear interpolation scheme while symbols correspond to the quadratic Lagrange polynomials. The difference cannot be appreciated on the scale of the diagram, and is always below the statistical accuracy of the data.

Starting from an already fully developed fluid velocity field in statistically steady conditions, five different populations of $N_{p}=300000$ particles each, with Stokes numbers $S t_{\eta}=0.1,0.5,1.0,5.0,10.0$, are initialized with random and homogeneous positions and velocities matching that of the local fluid. Samples for particle statistics corresponding to 120 independent snapshots separated in time by $2 S^{-1}$ are collected after an initial transient of $50 S^{-1}$. Discarding the initial transient is crucial to have results independent from the rather arbitrary initial state used to initialize the particles. We observe that, also under this respect, a statisticaly steady flow is mandatory to have well definite experimental conditions, especially in cases where the response of particle populations with different relaxation times are compared.

\section{Results \& discussion}

A visual impression of instantaneous particles configurations is provided in figure 3, where slices of the domain in selected coordinate planes are displayed for three different Stokes numbers. The typical particle distribution exhibits many voids, strongly correlated with high enstrophy, (7; 8), intertwined with thin "stretched" regions where particles concentrate. Clustering is specially manifest near $S t_{\eta}=1$, see the mid panel of the figure in comparison with top and bottom ones which show more even spreading. The typical void dimension, as caught by the eye, is larger at our largest Stokes number, $S t_{\eta}=10$. The ballistic limit, where particles follow their trajectories with no significant influence from the fluid and the expected spatial

distribution is homogeneous, is apparently still far away. In the opposite 
extreme case, passive tracers are recovered for vanishing $S t_{\eta}$, particles follow the fluid path, and, again, homogeneity is eventually restored. In fact, clustering still takes place, though at smaller scales, for the smallest Stokes number we have considered, consistently with theoretical arguments (10; 1) and numerical simulations (8; 9) for homogeneous and isotropic turbulence aimed at explaining droplets growth in clouds.

Despite of the mentioned similarities with isotropic flows, ours manifests specific features compelled by large scale anisotropy. The shear-induced orientation is apparent from the bottom-left/top-right alignment of particles sheets in the shear plane $x-y$, see the right panels of figure 3 . This behavior, clearly visible at $S t_{\eta}=1.0$, is still discernible in the other two cases. Since, apparently, the effect is strong and persistent, we are interested in putting forward suitable tools to evaluate the anisotropy of clustering. As we shall see, this is best done by extending in due form a line of analysis proved successful for the isotropic case.

The main statistical tool is the radial distribution function (RDF) of particle pairs $g(r)$ which is a function of radial distance $r$, see e.g. (33) where the RDF is dealt with for isotropic flows. The RDF, sometimes called correlation function, is defined as

$$
g(r)=\frac{1}{4 \pi r^{2}} \frac{d N_{r}}{d r} \frac{1}{n_{0}}
$$

where $n_{0}=0.5 N_{p}\left(N_{p}-1\right) / V_{0}$ is the density of pairs in the whole volume $V_{0}$ and $N_{r}$ is the number of pairs in a ball $\mathcal{B}_{r}$ of radius $r$.

The concept is easily extended to anisotropic cases by considering the number of pairs $d \mu_{r}=\nu_{r}(r, \hat{\mathbf{r}}) d \Omega$ contained in a spherical cone of radius $r$, with axis along the direction $\hat{\mathbf{r}}$ and solid angle $d \Omega$, see the sketch in the right panel of figure 1. By this definition the number of pairs in the ball $\mathcal{B}_{r}$ is $N_{r}=\int_{\Omega} \nu_{r} d \Omega$, hence $d N_{r} / d r=\int_{\Omega} d \nu_{r} / d r d \Omega$. We define the Angular Distribution Function (ADF) as

$$
g(r, \hat{\mathbf{r}})=\frac{1}{r^{2}} \frac{d \nu_{r}}{d r} \frac{1}{n_{0}},
$$

which retains information on the angular dependence of the distribution. The RDF is the spherical average of the ADF $g(r)=1 /(4 \pi) \int_{\Omega} g(r, \hat{\mathbf{r}}) d \Omega$ and it is shown in figure 4 for a few particle populations.

The behavior of the RDF near the origin, $g(r) \propto r^{-\alpha}$, is related to important geometrical features of the spatial distribution. Specifically, $\mathcal{D}_{2}=3-\alpha$ is the so-called correlation dimension of the multi-fractal measure associated with the particle density, (34). A positive $\alpha$ indicates the occurrence 
of small scale clustering. Its value is inferred from the slope near the origin in the log-log plots shown in figure 4, see the scaling behavior apparent in the range $r / \eta \in[.1: 1]$. From the figure, particles with $S t_{\eta} \sim 1$ exhibit maximum accumulation, i.e. the RDF diverges at a faster rate as $r$ is decreased, see also (24). The solid lines superimposed to the present data correspond to the scaling laws extracted at matching Stokes number by (8) in isotropic conditions. The agreement between our data and the isotropic ones is remarkable, showing that, even under strong shear, certain features of the clustering process may be universal.

Small scale clustering is not shared by heaviest particles. They show instead the saturation of the correlation function to a constant value $g(r) \simeq$ $g_{*}>1$ below a critical scale $\ell_{c}$, see e.g. the open squares in figure 4 with $\alpha \simeq 0$. This means that the number of pairs below $\ell_{c}$ is proportional to volume with an effective density $n_{*}=g_{*} n_{0}$ larger than its overall mean value $n_{0}$. By inspection of figure 4, for the heaviest particles (squares), the saturation occurs at $\ell_{c} \simeq 10 \eta$. The interpretation is that eddies with a time scale sufficiently smaller then particle Stokes time do not influence the clustering process. The saturation scale should then correspond to the size $\ell_{\min }$ of the smallest eddies able to aggregate particles. As an order of magnitude estimate, our data are consistent with the results given by (35) for the inertial range of isotropic turbulence, where the authors find $\ell_{\text {min }} / \eta=\left(S t_{\eta} / \beta_{\text {min }}\right)^{3 / 2}$ with $\beta_{\text {min }} \simeq 2$. Our data match this estimate also for the run at $S t_{\eta}=5$, where the saturation occurs close to the Kolmogorov scale. For lighter particles, clustering keeps on going below the Kolmogorov length and its lower limit cannot be interpreted by arguments which, tuned by experiment, are nevertheless taken from inertial range theory.

Concerning the large scale behavior, heavier particles apparently begin to show accumulation at larger scales. Each population is uniform with $g(r) \simeq 1$ at very large $r$. This trend is followed by the lightest particles down to $5 \eta$ below which they begin to follow the power-law. The RDF starts deviating from the uniform distribution $-g(r) \simeq 1$ - at a scale $\ell_{\max }$ which increases monotonically with the Stokes number, see. e.g. the range $r / \eta \in[20: 80]$. This is consistent with intuition, since the particle Stokes time progressively matches the eddy-turnover time of larger turbulent eddies. The data again agree reasonably well with the results of (35), which identify the range of time scales relevant to clustering as $\beta_{\max }<\tau_{p} / \tau(\ell)<\beta_{\text {min }}$ where $\tau(\ell) \propto \epsilon^{-1 / 3} \ell^{2 / 3}$ is the eddy turn over time at scale $\ell$. In terms of lengths, clustering starts to occur at $\min \left(\ell_{0}, \ell_{\max }\right)$ where $\ell_{0}$ is the integral scale and $\ell_{\max } / \eta=\left(S t_{\eta} / \beta_{\max }\right)^{3 / 2}\left(\beta_{\max } \simeq 0.1\right)$. Using this estimate, our 
heaviest particles are expected to begin accumulating at the integral scale $\ell_{0} / \eta \simeq 135$ while, e.g., the lightest ones at $\ell_{\max } / \eta \simeq 1$, which is not too far from the value $5 \eta$ we infer from the plot.

The RDF quantitatively confirms the overall impression gained from the visualizations of figure 3. The strong anisotropy apparent in those plots, however, needs a description in terms of the more complete ADF shown in figure 5 as a contour plot on the unit sphere for two separations $r$ and particles with $S t_{\eta}=1$. The ADF has been normalized with its average on the unit sphere, $g(r)$, in order to compare the relative anisotropy content of different scales. From figure 5, as separation decreases (i.e. moving from the right, $r=35 \eta$, to the left panel, $r=4 \eta$ ), the normalized ADF exhibits preferential clustering in directions consistent with those observed in the visualizations of figure 3 .

The ADF allows for a systematic evaluation of anisotropy in particle clustering. For given separation $r$, its angular dependence can be resolved in terms of spherical harmonics,

$$
g(r, \hat{\mathbf{r}})=\sum_{j=0}^{\infty} \sum_{m=-j}^{j} g_{j m}(r) Y_{j m}(\hat{\mathbf{r}}) .
$$

In this notation, the classical $\mathrm{RDF} g(r)$ is the projection of the $\mathrm{ADF}$ on the isotropic sector $j=0$, namely

$$
g_{00}(r) \equiv g(r)=\int_{\Omega} g(r, \hat{\mathbf{r}}) Y_{00}(\hat{\mathbf{r}}) d \Omega .
$$

This decomposition amounts in projecting our function on orthogonal subspaces invariant under rotations. Each sub-space is labeled by the index $j$ and it is spanned by $2 j+1$ base elements $Y_{j m}$. Growing levels of anisotropy are checked by increasing $j$, consistently with its geometrical meaning as number of zero crossings of $Y_{j m}$.

Figure 6 shows the normalized projections on sectors $j=2,4,6$, i.e. the normalized amplitudes $g_{j m} / g_{00}$, for the particle population with $S t_{\eta}=1$. For given $j$ most modes are negligible and the figure reports only those with significant signal level. The $(2,-2)$ mode provides the most significant contribution to the anisotropic component of $g(r, \hat{\mathbf{r}})$. The corresponding spherical harmonics, $Y_{2-2}$, roughly selects the intensity of the signal along the principal direction of the mean deformation tensor which corresponds to maximum straining and is inclined $45^{\circ}$ in the mean flow plane. $Y_{2-2}$ is negative in the first and third quadrant and positive in the others, thus explaining the negative sign of $g_{2-2}$ in figure 6. This description well captures 
the alignment of most thin particle clusters observed in the mid panel of figure 3 .

The signal content rapidly decreases with the order of the sector $j$ and the ADF is satisfactorily reconstructed-see decomposition (6)-using only the first few sectors with $j \leq 2$ as shown in bottom panels of figure 5. We conclude that the probability of finding two particles at separation $r=4 \eta$ along the main straining direction is $30 \%$ higher than in the perpendicular direction in the mean flow plane.

As discussed, the ADF provides a quantitative account of the anisotropy induced by the fluid velocity field on the disperse phase. As shown below, it can be effectively used to parameterize the level of anisotropy through the scales in terms of the Stokes number.

The plot of figure 7 gives the normalized amplitude of the most energetic anisotropic mode in absolute value $-\left|g_{2-2}\right| / g_{00}$-for our set of Stokes numbers $S t_{\eta}=10,5,1,0.5,0.1$ ranging from heavy to light particles. Focusing on the heaviest particles, $S t_{\eta}=10,5$, the relative amplitude of the strongest anisotropic mode first increases towards the small scales to reach a maximum at $r \sim \ell_{c}$. Below this scale the anisotropy level decreases, until the very small scales become essentially isotropic. Connecting this result with the previous discussion concerning the saturation of the RDF $g_{00}$, we conclude that the heaviest particles show a regular concentration at scales smaller than $\ell_{c}$ where the distribution recovers isotropy.

Particles with smaller Stokes numbers behave in an entirely different way. The anisotropy, as measured by the ratio $g_{2-2} / g_{00}$, substantially increases to saturate at small scales close to Kolmogorov length. It keeps an almost constant value below $\eta$. In other words, the clustering process maintains its anisotropic features even below the dissipative scale for sufficiently small Stokes number particles. Remind that the overall clustering process described by $g_{00}$ is here characterized by a singular exponent $\alpha$. The saturation observed on the ratio $g_{2-2} / g_{00}$ implies that the dominating anisotropic contribution inherits the same behavior, $g_{2-2} \propto r^{-\alpha}$.

\section{Final comments}

We have provided evidence that large scale shear induces preferential orientation on the patterns a disperse phase of small inertial particles forms in turbulence. The effect is indirect: The shear imprints anisotropy on velocity fluctuations which, in turn, arrange particle configurations in directionally biased clusters. 
Recently it became increasingly clear that the multi-scale nature of the velocity field is crucial in explaining most features of particles distributions. Typically, a range of eddies exists able to break spatial homogeneity of particles configurations. The particles segregate and their pattern shows local concentrations and voids on length scales correlated with those of the inducing eddies. The range of scales of the inducing eddies is determined by the Stokes time of the particles, and moves from integral down to Kolmogorov length with reducing the relaxation time. For light particles, clustering reaches down Kolmogorov scale, leading to a singularity in the radial distribution function. The exponent of the singularity is a measure of its intensity. All these features, originally found in experiments and numerical simulations of isotropic turbulence, are also present in our anisotropic fields. They are thus generic aspects of the clustering process which seem independent of the specific geometry of the forcing.

At a qualitative level, the specific characteristics of clustering under anisotropic advection consists of preferential orientation of the particle patterns. In order to quantify the new scale-dependent features added by the non-trivial geometry of the forcing, we have introduced the concept of angular distribution function. It can be understood as a generalization of the previous radial distribution function, to which it reduces by averaging on the unit sphere, i.e. by performing the isotropic projection. This quantitative tool has led to our most unexpected finding: The advecting field anisotropy, known to be confined to the large scales, affects the singular, small scale, clustering process. In fact, anisotropy results into a strong directionality of the probability to find a couple of particles at viscous scale separation, with $30 \%$ variations on the solid angle easily observed.

Technically, for Stokes number order unity, the anisotropic component of the angular distribution function diverges at small scales with singularity exponent comparable to that found in the isotropic projection (RDF). For very small Stokes number, we cannot even exclude that the singularity exponent of the strongest anisotropic sector may even exceed that of the radial distribution function. Conversely, heavy particles appear to preferentially concentrate on finite sized patches endowed with a range of multi-scale and shear oriented features, with finest scales more or less evenly and isotropically distributed.

The geometrical properties of patterns of inertial particles differ considerably from that one could naively guess from velocity fluctuations. Recent findings, extending somehow a number of previous results on shear induced anisotropy (19, 36, 37, 38, 20), show that velocity fluctuations manifest two neatly distinct ranges, one dominated by the production of turbulent 
kinetic energy above the shear scale $L_{s}$, the other, corresponding to the classical inertial range of Kolmogorov theory below. In the two ranges, velocity fluctuations display different isotropy recovery rates, a smaller one in the production range, a larger one in the inertial transfer range between $L_{s}$ and the viscous scale $\eta,(22 ; 21)$. Actually, concerning the velocity field, isotropy is matter of fact always recovered at dissipative scales, provided the scale separation $L_{s} / \eta$ is large enough, i.e. the local Reynolds number (TaylorReynolds number, or, equivalently for wall bounded flows the wall normal distance in inner units, $y^{+}=y \sqrt{\tau_{w} / \rho} / \nu$, with $\tau_{w}$ the wall shear stress) is sufficiently large. This condition is violated in an essential way only very close to solid walls in wall-bounded turbulence (small $y^{+}$).

The anisotropy in the particle configurations depends strongly on the properties of the advecting velocity field. However, despite of the isotropy recovery of the velocity field, isotropy may never be recovered in the small scales of the clusters, as it happens at small Stokes number. Actually, in the range from $L_{s}$ to $\eta$ clustering of light inertial particles shows a substantial increase of directionality.

From our results at moderate Reynolds number one can conjecture the behavior of the clusters at high Reynolds numbers. In principle the intermittency of the turbulent field may induce a dependence on Reynolds number. In fact, as shown in (8) for isotropic flows, the fractal properties of particle distributions depend at most weakly on Reynolds number and strongly on the Stokes number. For given geometry of the external forcing, i.e. fixing the integral and the shear scale, and at given particle Stokes time, the increase of the Reynolds number corresponds to increasing the Stokes number based on Kolmogorov time, $S t_{\eta}$. Given the weak dependence on Reynolds number, this is somehow equivalent to reading figs. 4 and 7 by successively moving from light to heavy particles. Along the process, we infer the saturation at small scales of the radial distribution function $\left(S t_{\eta} \gg 1\right.$, fig. 4) and the small scale isotropy of the clusters (fig. 7). Clustering is confined to the intermediate scales where it shows high levels of anisotropy. This means that, in the limit of large Reynolds number, any particles population should be organized in anisotropic finite-sized patches which are eventually uniform and isotropic in their finest scales. We stress once more that an intermediate range of scales always exists, however, where a multi-scale aggregation process takes place with a substantial directionality of the clusters.

On the other hand, at finite Reynolds number, sufficiently small particlessmall relaxation time-will always show small scale clustering, in the sense of a singularity in the radial distribution function. In this case anisotropy may persist below Kolmogorov scale, as described by the angular distribution 
function at small separations for the lightest populations in our simulations. This finite Reynolds number effect becomes extremely important in the near wall region of turbulent wall bounded flows, where the local Reynolds number constructed with the distance from the wall is not huge and the velocity field is meanwhile strongly anisotropic.

The issuing anisotropy of the fine scales of particles clusters will then have a significant impact on phenomena of collision, aggregation of dusts into larger particles, evaporation/condensation rate of droplets in pipe lines and a number of other physically and technologically significant contexts.

A final comment concerns the extension of the present results to the near wall region of wall bounded flows. As shown by recent results on the scaleby-scale statistics of the velocity field, e.g. energy transfer across scales, spectral distribution of turbulent kinetic energy production, intermittency and anisotropy, the homogeneous shear flow reproduces the essential features observed in the wall region, despite of significant differences in the large scale geometry of the two systems. However, particles in the wall region are strongly affected by turbophoresis which is a predominant effect associated with inhomogeneity. In wall flows particle segregation is controlled by the two concurrent processes of small scale anisotropic clustering and of accumulation at the wall. Clearly, the focus of the present paper is on the former one, leaving the combined analysis of the two effects for future investigations. 


\section{References}

[1] G. Falkovich, A. Fouxon, and M. Stepanov. Acceleration of rain initiation by cloud turbulence. Nature (London), 419:151, 2002.

[2] R. A. Shaw. Particle-turbulence interactions in atmospheric clouds. Ann. Rev. Fluid Mech., 35:183, 2003.

[3] A. Bracco, P.H. Chavanis, A. Provenzale, and E.A. Spiegel. Particle aggregation in a turbulent keplerian flow. Phys. Fluids, 11:2280, 1999.

[4] G. Károlyi, Á. Péntek, I. Scheuring, T. Tél, and Z. Toroczkai. Chaotic flow: The physics of species coexistence. Proc. Natl. Acad. Sci., 97:13661, 2000 .

[5] D. W. I. Rouson and J. K. Eaton. On the preferential concentration of solid particles in turbulent channel flow. J. Fluid. Mech., 428:149, 2001.

[6] C. Marchioli and A. Soldati. Mechanisms for particle transfer and segregation in a turbulent boundary layer. J. Fluid. Mech., 468:283, 2002.

[7] K. D. Squires and J. K. Eaton. Preferential concentration of particles by turbulence. Phys. Fluids A, 3:1169, 1991.

[8] J. Bec, L. Biferale, M. Cencini, A. Lanotte, S. Musacchio, and F. Toschi. Heavy particle concentration in turbulence at dissipative and inertial scales. Phys. Rev. Lett., 98(8):084502, 2007.

[9] W. C. Reade and L. R. Collins. Effect of preferential concentration on turbulent collision rates. Phys. Fluids, 12:2530, 2002.

[10] E. Balkovsky, G. Falkovich, and A. Fouxon. Intermittent distribution of inertial particles in turbulent flows. Phys. Rev. Lett., 86:2790, 2001.

[11] M.W. Reeks. The transport of discrete particles in inhomogeneous turbulence. J. Aerosol Sci., 14(6):729-739, 1983.

[12] M. Righetti and G.P. Romano. Particle-fluid interaction in a plane near-wall turbulent flow. J. Fluid. Mech., 505:93-121, 2004.

[13] D. Kaftori, G. Hetsroni, and S. Benerjee. Particle behavior in the turbulent boundary layer. part i: Motion, deposition, and entrainment. Phys. Fluids, 7(5):1095-1106, 1995. 
[14] D. Kaftori, G. Hetsroni, and S. Benerjee. Particle behavior in the turbulent boundary layer. velocity and distribution profiles. Phys. Fluids, 7(5):1107-1121, 1995.

[15] L.M. Portela, P. Cota, and R.V.A. Oliemans. Numerical study of thr near-wall behaviour of particles in turbulent pipe flow. Powder Technology, 125:149-157, 2002.

[16] J.W. Brooke, K. Kontomaris, T.J. Hanratty, and J.B. McLaughlin. Turbulent deposition and trapping of aerosols at wall. Phys. Fluids, A 6(4):825-834, 1992.

[17] X. Shen and Z. Warhaft. The anisotropy of small scale structures in high reynolds number $\left(r e_{\lambda} \sim 1000\right)$ turbulent shear flow. Phys. Fluids, 12(11):2976-2989, 2000.

[18] P. Gualtieri, C.M. Casciola, R. Benzi, and R. Piva. Preservation of statistical properties in large eddy simulation of shear turbulence. $J$. Fluid. Mech., 592:471-494, 2007.

[19] S. Corrsin. Local isotropy in turbulent shear flow. Naca Research Memorandum, RM 58B11:1, 1958.

[20] Z. Warhaft and X. Shen. On higher order mixed structure functions in laboratory shear flow. Phys. Fluids, 14:2432, 2002.

[21] B. Jacob, C. M. Casciola, A. Talamelli, and P. H. Alfredsson. Scaling of mixed structure functions in turbulent boundary layers. Phys. Fluids, 20:045101, 2008.

[22] C. M. Casciola, P. Gualtieri, B. Jacob, and R. Piva. The residual anisotropy of small scales in high shear turbulence. Phys. Fluids, 19:101704, 2007.

[23] L. Biferale and I. Procaccia. Anisotropy in turbulent flows and in turbulent transport. Phys. Rep., 414:43, 2005.

[24] B. Shotorban and S. Balachandar. Particle concentration in homogeneous shear turbulence simulated via lagrangian and equilibrium eulerian approaches. Phys. Fluids, 18:065105, 2006.

[25] A. M. Ahmed and S. Elghobashi. On the mechanism of modifying the structure of turbulent homogeneous shear flows by dispersed particles. Phys. Fluids, 12:2906, 2000. 
[26] A. M. Ahmed and S. Elghobashi. Direct numerical simulation of particle dispersion in homogeneous turbulent shear flows. Phys. Fluids, 13:3346, 2001.

[27] B. Shotorban, F. Mashayek, and R. V. R. Pandya. Temperature statistics in particle-laden turbulent homogeneous shear flow. Int. J. Multiphase Flow, 29:1333, 2003.

[28] R.S. Rogallo. Numerical experiments in homogeneous turbulence. Nasa $T-M, 81315,1981$.

[29] P. Gualtieri, C. M. Casciola, R. Benzi, G. Amati, and R. Piva. Scaling laws and intermittency in homogeneous shear flow. Phys. Fluids, 14:583, 2002.

[30] P.K. Yeung and S.B. Pope. An algorithm for tracking fluid particles in numerical simulations of homogeneous turbulence. J. Comp. Phys., 79:373-416, 1988.

[31] S. Balachandar and M.R. Maxey. Methods for evaluating fluid velocities in spectral simulations of turbulence. J. Comp. Phys., 83:96-125, 1989.

[32] M. R. Maxey and J. J. Riley. Equation of motion for a small rigid sphere in a nonuniform flow. Phys. Fluids, 26:2437, 1983.

[33] S. Sundaram and L. R. Collins. Collision statistics in an isotropic particle-laden turbulent suspension. part 1. direct numerical simulations. J. Fluid. Mech., 335:75, 1997.

[34] P. Grassberger and I. Procaccia. Characterization of strange attractors. Phys. Rev. Lett., 50:346, 1983.

[35] H. Yoshimoto and S. Goto. Self-similar clustering of inertial particles in homogeneous turbulence. J. Fluid. Mech., 577:275-286, 2007.

[36] M.S. Uberoi. Equipartition of energy and local isotropy in turbulent flows. J. Appl. Phys., 28(10):1165-1170, 1957.

[37] W.K. George and H.J. Hussein. Locally axisymmetric turbulence. J. Fluid. Mech., 233:1-23, 1991.

[38] R.A. Antonia and J. Kim. A numerical study of local isotropy of turbulence. Phys. Fluids, 6(2):834-841, 1994. 

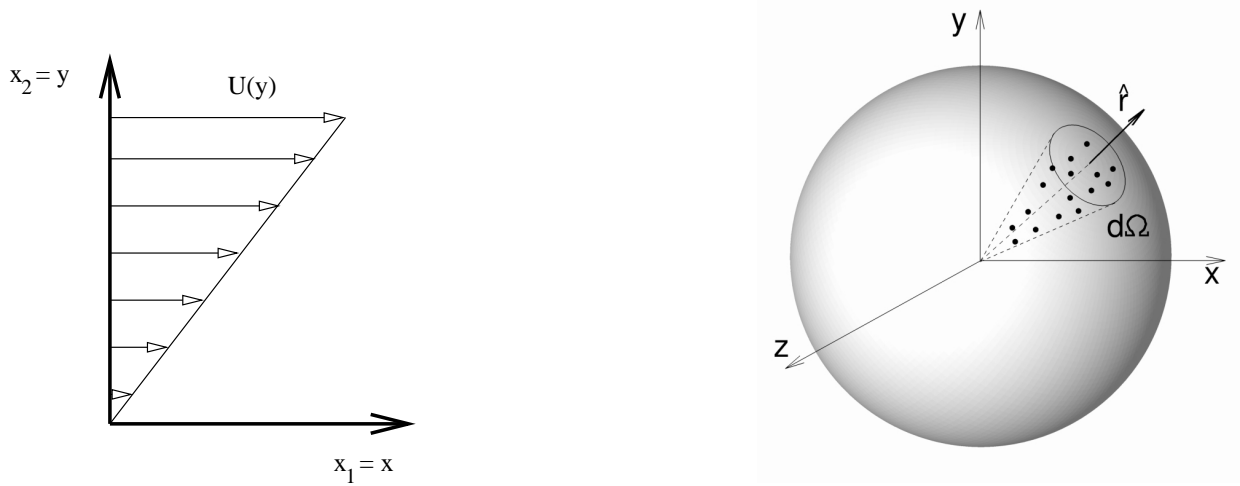

Figure 1: Left: sketch of the shear flow and nomenclature: the mean flow $U(y)$, in the $x \equiv x_{1}$ direction, is a function of $y \equiv x_{2}$, with $z \equiv x_{3}$. For a linear mean profile, the shear rate $S=d U / d y$ is constant. Right: sketch of the spherical cone of amplitude $d \Omega$ in direction $\hat{\mathbf{r}}$. 

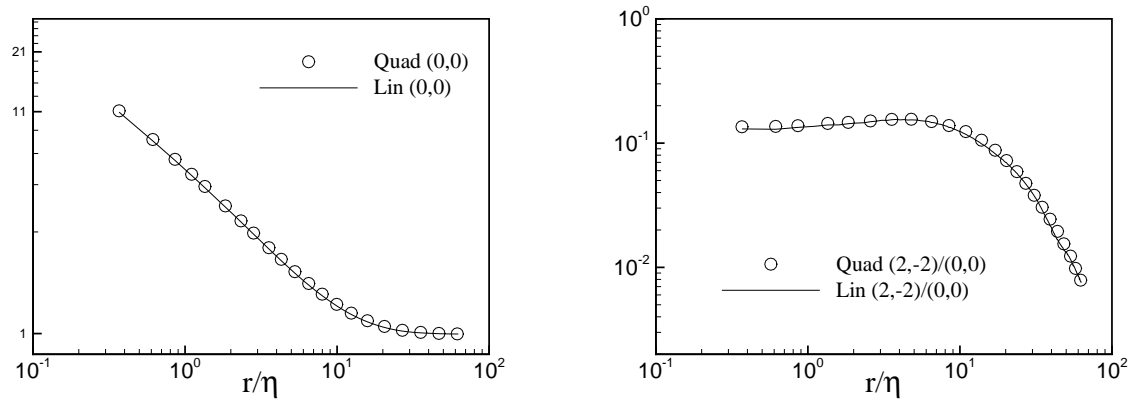

Figure 2: DNS of particle laden homogeneous shear flow $\left(R e_{\lambda}=60, S^{*}=7\right.$, $\left.S t_{\eta}=1\right)$, in a $4 \pi \times 2 \pi \times 2 \pi$ computational box with $192 \times 192 \times 96$ collocations points corresponding to $K_{\max } \eta=3$. The disperse phase is computed by using two different schemes to interpolate the fluid velocity at particle positions, namely linear interpolation (solid line) and quadratic Lagrange polynomials (symbols). The statistical observables shown in the plots are defined in $\S 3$. Left panel: $g_{00}(r)$, projection of the ADF in the isotropic sector. Right panel: $\left|g_{2-2}\right| / g_{00}$, normalized most energetic anisotropic component of the ADF. 

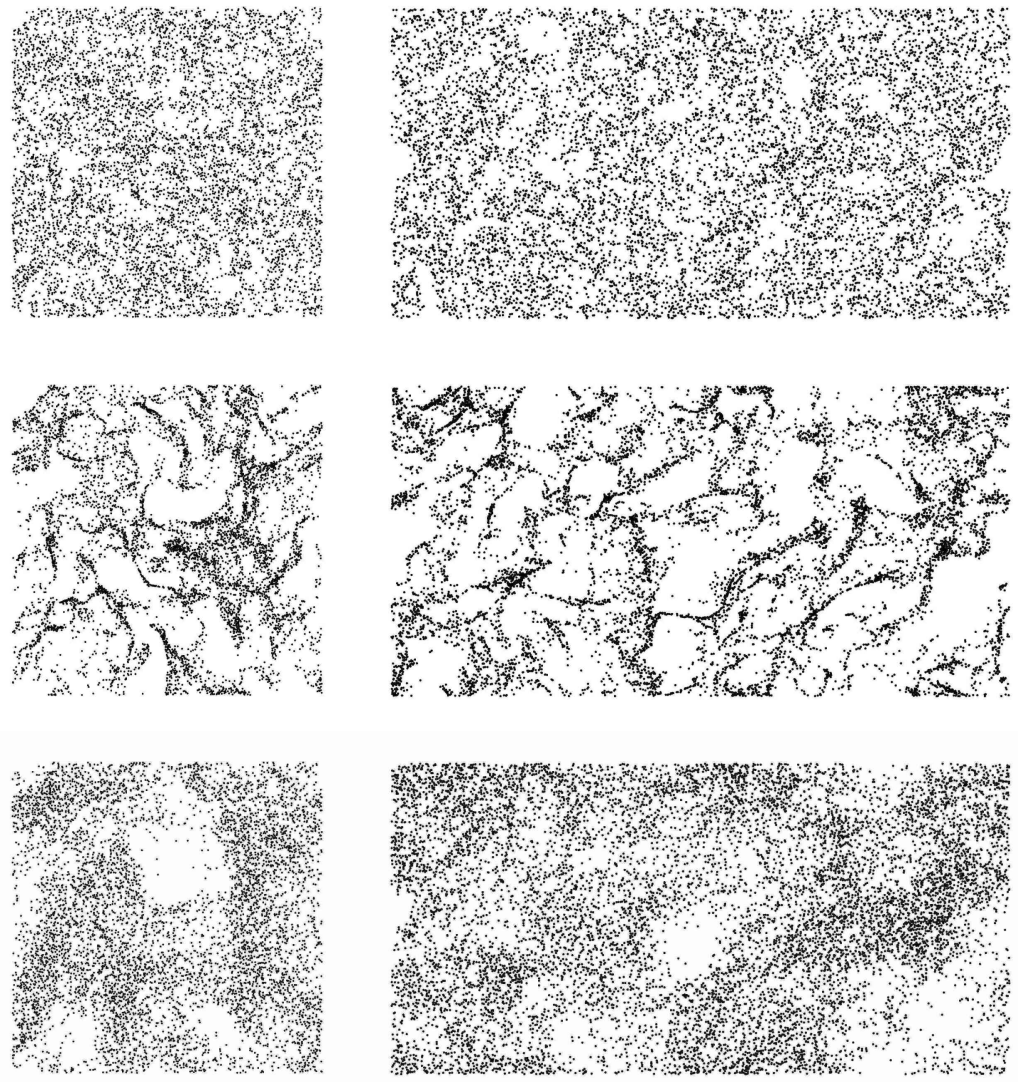

Figure 3: Snapshots of particle positions for increasing Stokes number, from top to bottom: $S t_{\eta}=0.1,1,10$ respectively. Left column thin slice in the $y-z$ plane; right column slice in the $x-y$ plane. The slice thickness is of the order of a few Kolmogorov scales. 


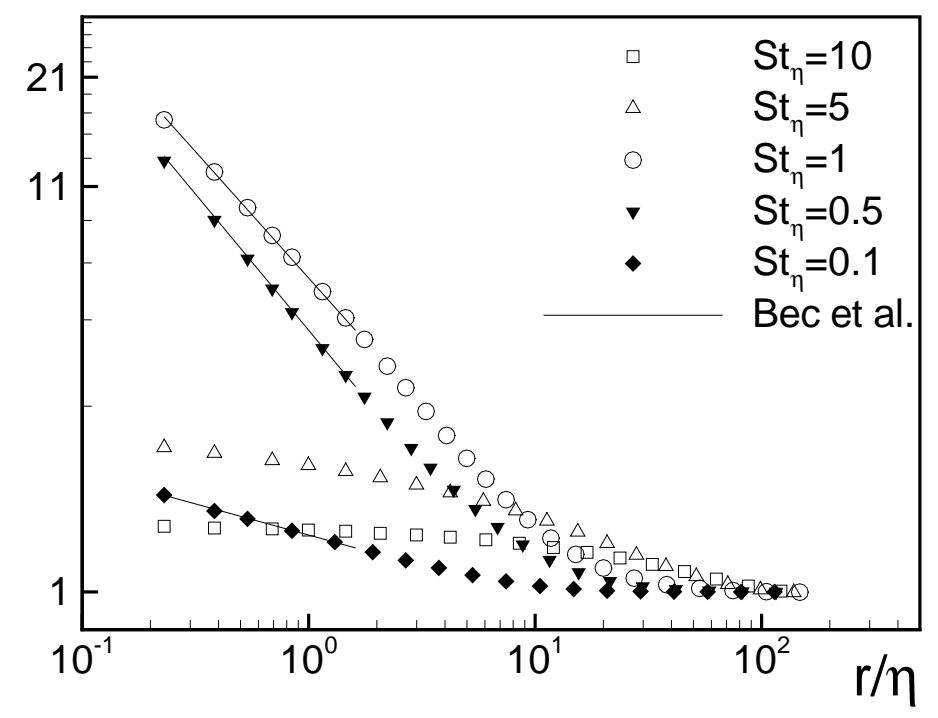

Figure 4: Radial distribution function $v s$ separation, for different Stokes number. 

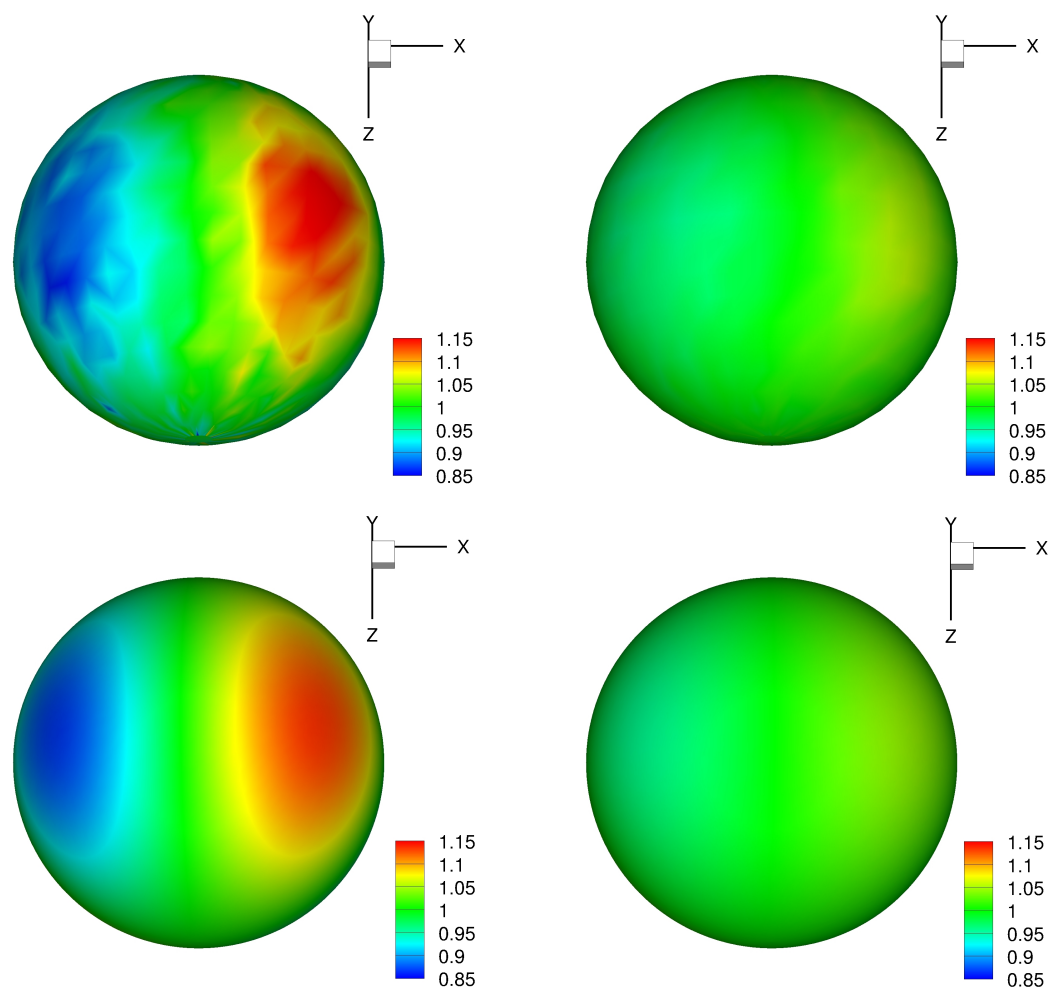

Figure 5: Top panels: Angular Distribution Function (ADF) giving the probability per unit solid angle to find a couple of particle at fixed distance $|\mathbf{r}|$. Left panel ADF computed at separation $4 \eta$; right panel $35 \eta$. Data for $S t_{\eta}=1$. Bottom panels: Estimate of ADF by using only the isotropic sector and the $j=2$ sector. 


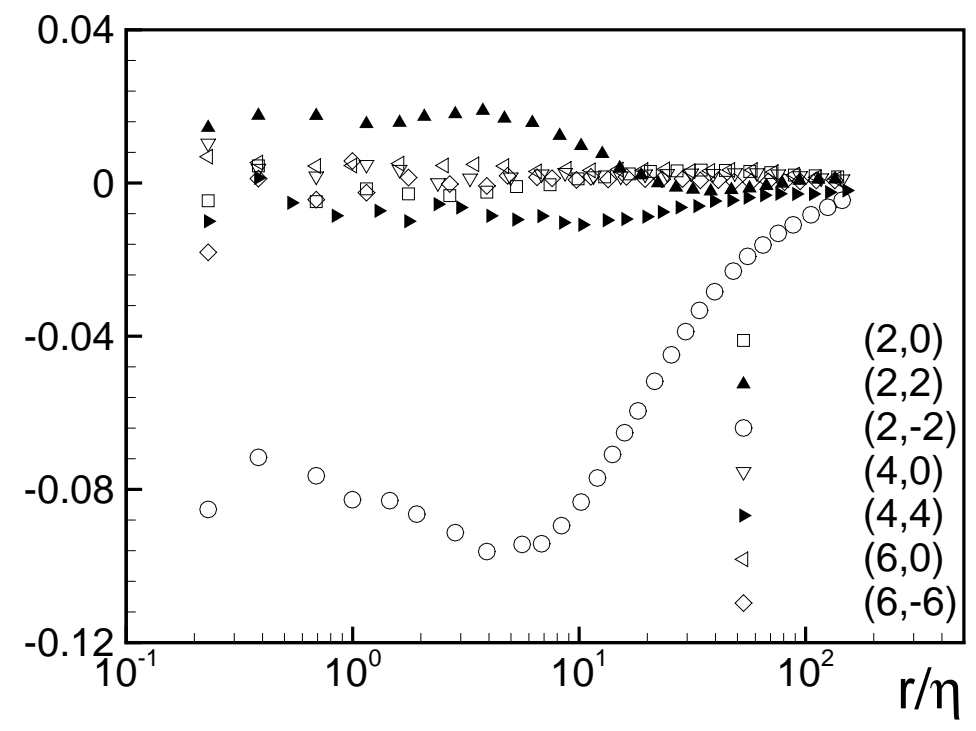

Figure 6: Projection of ADF on the different anisotropic sectors of spherical harmonics normalized by the projection of the isotropic sector (RDF) as a function of separation. Data for $S t_{\eta}=1$. 


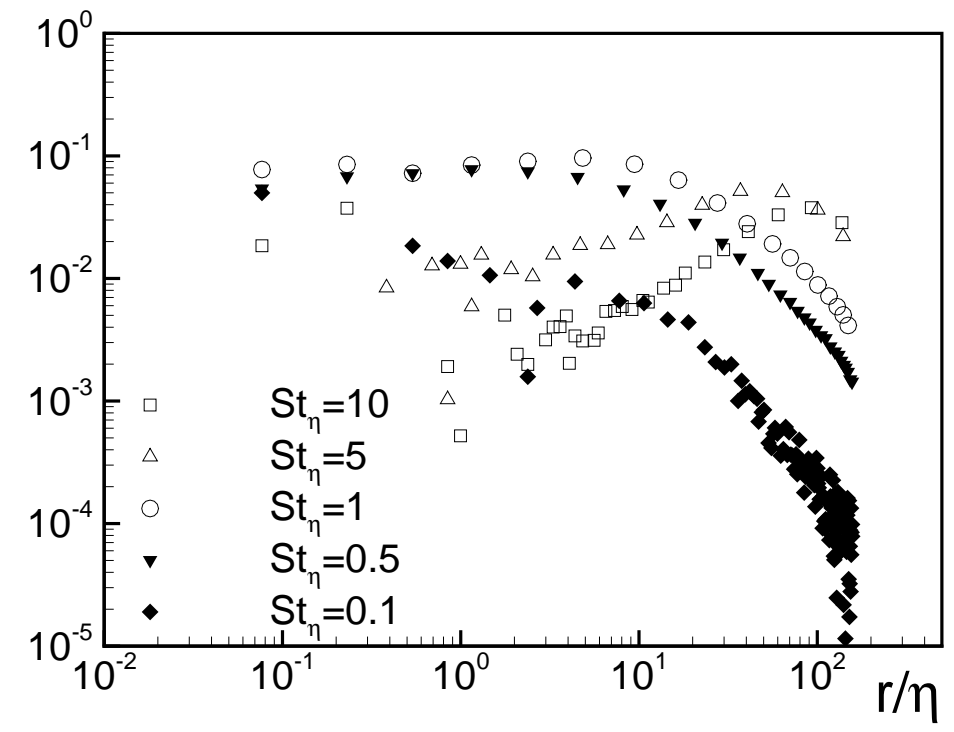

Figure 7: Ratio between the most energetic anisotropic sector $(2,-2)$ normalized by isotropic sector as a function of separation for different Stokes number. 